\title{
La fleur de lotus ou Nelumbo nucifera dans les mosaïques gréco- romaines
}

\section{Greko-Romen Mozaiklerinde Lotus Çiçeği veya Nelumbo Nucifera}

Véronique VASSAL*

(Received 31 January 2021, accepted after revision 07 September 2021)

\begin{abstract}
The Lotus Flower or Nelumbo Nucifera in Greco-Roman Mosaics

Numerous mosaics from the Hellenistic and imperial periods with Nilotic decoration have been recorded, both in the West and in the East. Almost all of them have a vegetal decoration illustrating the flora of the Nile. Among this lush vegetation, the lotus is reproduced in various forms and it is worth studying some of these representations in detail. Some examples seem to be part of an Alexandrian tradition, but particularities may evoke the world of Pharaonic Egypt. We have endeavoured to highlight how the expression of these traditions in iconography has manifested itself as well as in the treatment of the decoration.

In the first instance, we thought it would be useful to go back over a few notions of botany and the characteristics of water lilies in Egypt. Different species are very similar, which leads to multiple risks of misunderstanding when using iconographic or literary sources.

In the second instance, we have tried to show how Greco-Roman mosaic artists rendered the different stages of the growth of these flowers through their art. The naturalistic aspect, very present in Hellenistic mosaics, seems to have changed during the imperial period. Does the lotus retain something of the earlier floral vision, when it adorned the pavements of the Roman provinces?

In the House of the Fauna in Pompeii, the mosaic of the Battle of Alexander and Darius decorated an exedra. The threshold, delimited by two Corinthian columns, was decorated with a mosaic consisting of several panels depicting scenes of life on the Nile, dating from the end of the $2^{\text {nd }}$ century BC. These have been widely described and we have only focused here on the representation of lotus flowers. The illustration seems to visually describe the different stages of the flower's life cycle. This is, in particular, the case as regards the Barberini mosaic in Préneste. The lotus sometimes appears from the front with its petals outspread. The nelumbo then consists of several corollae, the petals of which display shades of colour ranging from dark pink to light pink. This treatment is reminiscent of the fragment from the mosaic of the Canope baths, where a large nelumbo with two corollae unfolds in a circular composition.
\end{abstract}

Keywords: Mosaics, Nile, lotus, Nelumbo, duck.

$\ddot{\mathrm{O} z}$

Hem Batı'da hem de Doğu'da, Helenistik ve Imparatorluk dönemlerine ait çok sayıda Nil konulu mozaik kaydedilmiştir. Hemen hemen hepsinde Nil'in florasını gösteren bitkisel bir bezeme vardır. Bu yemyeşil bitki örtüsü arasında nilüfer çeşitli şekillerde yeniden üretilmektedir ve bu temsillerden bazılarını ayrıntılı olarak incelemeye değer. Bazı örnekler İskenderiye geleneğinin bir parçası gibi görünmektedir, ancak bunların özellikleri Firavun dönemi Mısır dünyasını çağrıştırıyor olabilir. Bu geleneklerin ikonografideki ifadesinin, süslemenin işlenmesinde olduğu kadar kendini nasıl gösterdiğini de vurgulamaya çalıştık.

\footnotetext{
* Véronique Vassal, chercheur associé, Equipe ArScAn-Archéologie du Monde grec et systèmes d'information UMR 7041 Archéologies et Sciences de l'Antiquité CNRS - Université de Paris I - Université de Paris Ouest-Nanterre - Ministère de la Culture Maison René Ginouvès. 157, rue de Charenton 75012 Paris, France. (iD https://orcid.org/0000-0002-6320-3337. E-mail: v.vassal@gmail.com
} 
İlk önce, birkaç botanik kavramına ve Mısır'daki nilüferlerin özelliklerine geri dönmenin faydalı olacağını düşündük. Farklı türler birbirine çok benzemektedir, bu da ikonografik veya edebi kaynakları kullanırken birden fazla yanlış anlama riskine yol açmaktadır.

İkinci etapta ise, Yunan - Roma mozaik sanatçılarının bu çiçeklerin gelişiminin farklı aşamalarını sanatlarıyla nasıl işlediklerini göstermeye çalıştık. Helenistik mozaiklerde çokça görülen natüralist husus, imparatorluk döneminde değişmiş görünmektedir. Lotus, Roma kentlerinin döşemelerini süslerken daha önceki çiçek görünümünden bir şeyler koruyor mu?

Pompeii'deki Faun Evi'nde, İskender ve Darius Savaşı'nın mozaiği bir eksedrayı süslemektedir. İki korinth sütunuyla sinırlanan eşik, IÖ 2. yüzyllın sonlarından kalma Nil'deki yaşam sahnelerini gösteren birkaç panelden oluşan bir mozaikle süslenmiştir. Bunlar geniş çapta tanımlanmıştır ve biz burada yalnızca lotus çiçeklerinin temsiline odaklandık. Çizim, çiçeğin yaşam döngüsünün farklı aşamalarını görsel olarak tanımlıyor gibi görünmektedir. Bu durum, özellikle Préneste'deki Barberini Mozaiği için geçerlidir. Lotus bazen önden yaprakları yayılmış hâlde görünmektedir. Lotus çiçeği (nelumbo) daha sonra, yaprakları koyu pembeden açık pembeye kadar değişen renk tonları sergileyen birkaç taçtan oluşur. Bu işleme, iki taçlı büyük bir lotus çiçeğinin dairesel bir kompozisyon içinde açıldığı Canope Hamamlarının mozaiklerinden gelen bir parçayı andırmaktadır.

Anahtar Kelimeler: Mozaikler, Nil, lotus, Nilüfer Çiçeği, ördek.

De nombreuses mosaïques d'époque hellénistique et impériale à décor nilotique ont été répertoriées, aussi bien en Occident qu'en Orient. Presque toutes ont un décor végétal qui illustre la flore du Nil. Parmi cette végétation luxuriante, le lotus est reproduit sous différentes formes. Nous nous proposons d'étudier précisément quelques-unes de ces représentations. Certains exemples semblent s'inscrire dans une tradition alexandrine, mais des particularités peuvent évoquer le monde de l'Égypte pharaonique. Nous nous attacherons à mettre en évidence quelques-unes des manifestations de l'expression de ces traditions dans l'iconographie comme dans le traitement du décor.

\section{Les lotus et les nénuphars en botanique}

Revenons tout d'abord sur quelques notions de botanique et sur les caractéristiques des nymphéas en Égypte. En effet, les nénuphars ont été classés dans l'ordre des Nymphaeales et dans la famille des Nymphéacées que l'on nomme improprement « lotus ». Le lotus bleu ${ }^{1}$, Nymphea caerulea Sav., possède des pétales fins se terminant en pointes. Les feuilles sont rondes, plates et hydrofuges. Les nombreux documents égyptiens - stèles funéraires, bas-reliefs, peintures de tombe - attestent que cette fleur occupait une place importante. Le lotus bleu flottait sur les eaux ; s'ouvrant dès les premiers rayons du soleil, il se refermait le soir venu et de sa fleur épanouie, s'échappait un parfum délicat. Particulièrement apprécié pour ses qualités sédatives et médicinales ${ }^{2}$, il était également utilisé pour orner les parures funéraires ${ }^{3}$, mais symbolisait aussi la Haute-Égypte (Janick 2002 : 1, 3-4). Le Nymphea caerulea ne doit pas être confondu avec le Nelumbium speciosum Willd connu également sous le nom de Nelumbo nucifera Gaertner plante aquatique vivace rhizomateuse qui pousse dans les eaux stagnantes et se classe dans la famille des Nélumbonacées. Il s'agit du seul lotus rose probablement introduit en Égypte de l'Inde 4 .

Les racines sont appelées des rhizomes, des sortes de tubercules de nature

1 Seulement mentionné par Athen. deipn. XV, 677 : « Le lotus qui là porte le nom Antinoeios, ... pousse dans les marais pendant l'été. Il est de deux couleurs. Un ressemble à la rose et c'est avec lui qu'est tressée la guirlande... L'autre est appelé lotus et sa couleur est bleue ».

2 Apomorphine : dérivé de la morphine.

3 Vers 1883, le botaniste allemand Georg Schweinfurth étudia les guirlandes de fleurs déposées sur la momie de Ramsès II. Il constitua des herbiers dont six planches sont aujourd'hui conservées au Muséum d'histoire naturelle de Paris. Ces dernières attestent de l'utilisation des fleurs de Nymphaea caerulea Sav. sur des parures funéraires. Voir Morat 2004 : 176-180.

4 Espèce venant d'Inde, voir Li et al. 2014 : 288. Dans les textes anciens, il est parfois confondu avec le colocasia (Colocasia esculenta), voir Whitehouse 2003 : 252-259 ; Grimaldi 2018 : 6. 
spongieuse d'une dizaine de centimètres de long. Ces rhizomes sont liés entre eux par un petit renflement qui porte le nom de nodosité. De ces structures naissent les tiges aériennes ou pédoncules qui vont porter des fleurs de couleur rose, des feuilles planes, dont le limbe flotte à la surface de l'eau, et d'autres orbiculaires à bords relevés (Fig. 1 a-b-c-d-e).

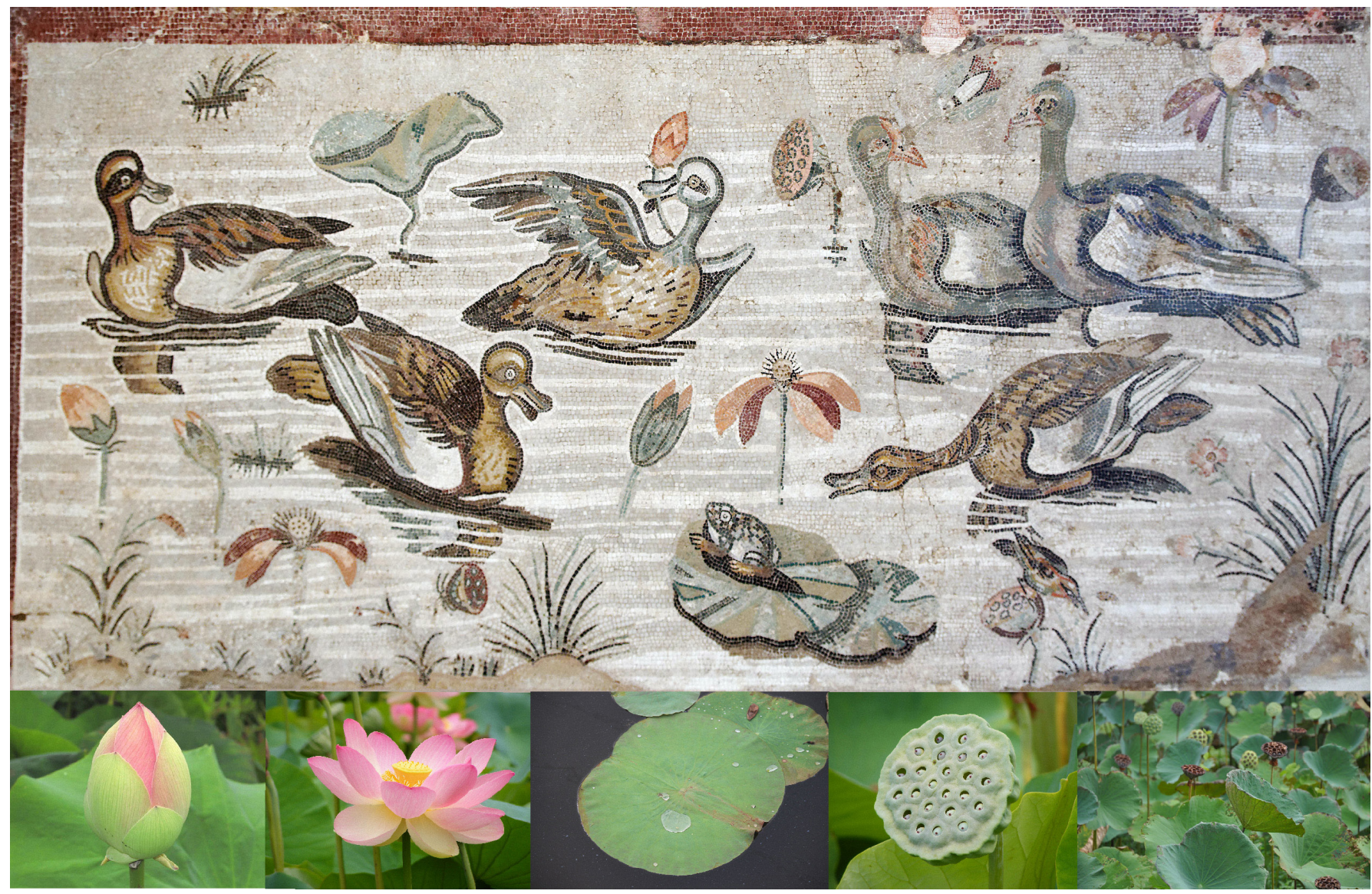

Figure 1

Pompéi, Maison du Faune, détail de l'un des panneaux devant l'exèdre de la mosaïque d'Alexandre et Darius. Figure $1 \mathrm{a}-\mathrm{b}-\mathrm{c}-\mathrm{d}$ : Nelumbo nucifera a-lotus en bouton ; b-fleur épanouie ;

c-feuille plane à la surface de l'eau ; d-réceptacle ;

e-champs de lotus et feuilles orbiculaires. Photo : V. Vassal.
Ces différentes espèces se ressemblent énormément, ce qui conduit à de multiples risques de méprise lorsque l'on utilise les sources iconographiques ou littéraires.

Le lotus apparaît sur les décors de peintures grecques, comme à Mieza dans la tombe des palmettes, vers la fin du IVe et le début du IIII siècle avant J.-C. (Fig. 2). En architecture, il décore la base d'une colonne du temple de Didymes datée vers 300 avant J.-C. (Wiegand 1941) (Fig. 3), enfin, sur les mosaïques, la fleur se manifeste en compositions linéaires alternant avec des palmettes comme sur la bordure de la mosaïque de l'amazonomachie de Pella, datée de la fin du IV siècle avant J.-C. (Ginouvès 1993 : 128-129, 132-136 fig. 113, 118) (Fig. 4). Les lotus sont stylisés et composés de plusieurs calices. Selon John Boardman (1998: 16) : «Lotus friezes and palmette friezes were common in Mesopotamia centuries before. Combining them in a botanically incorrect manner in one frieze was a Greek conceit which became enormously popular through the whole Greek world from the later seventh century B.C. ". En dehors de ces compositions, on remarque la présence du nelumbo nucifera sur les scènes nilotiques des périodes hellénistiques et romaines, souvent associé à une faune (canards, oies, hippopotames...) ou à des pygmées (Lancha 1980 ; Versluys - Meyboom 2000 ; Versluys 2002 ; Guimier-Sorbets 2009 : 664-663 ; 2013 :141-154 ; 2019b : 101110).

Nous tenterons de montrer ici, comment les mosaïstes gréco-romains rendaient par leur art les différentes étapes de la croissance de ces fleurs. 


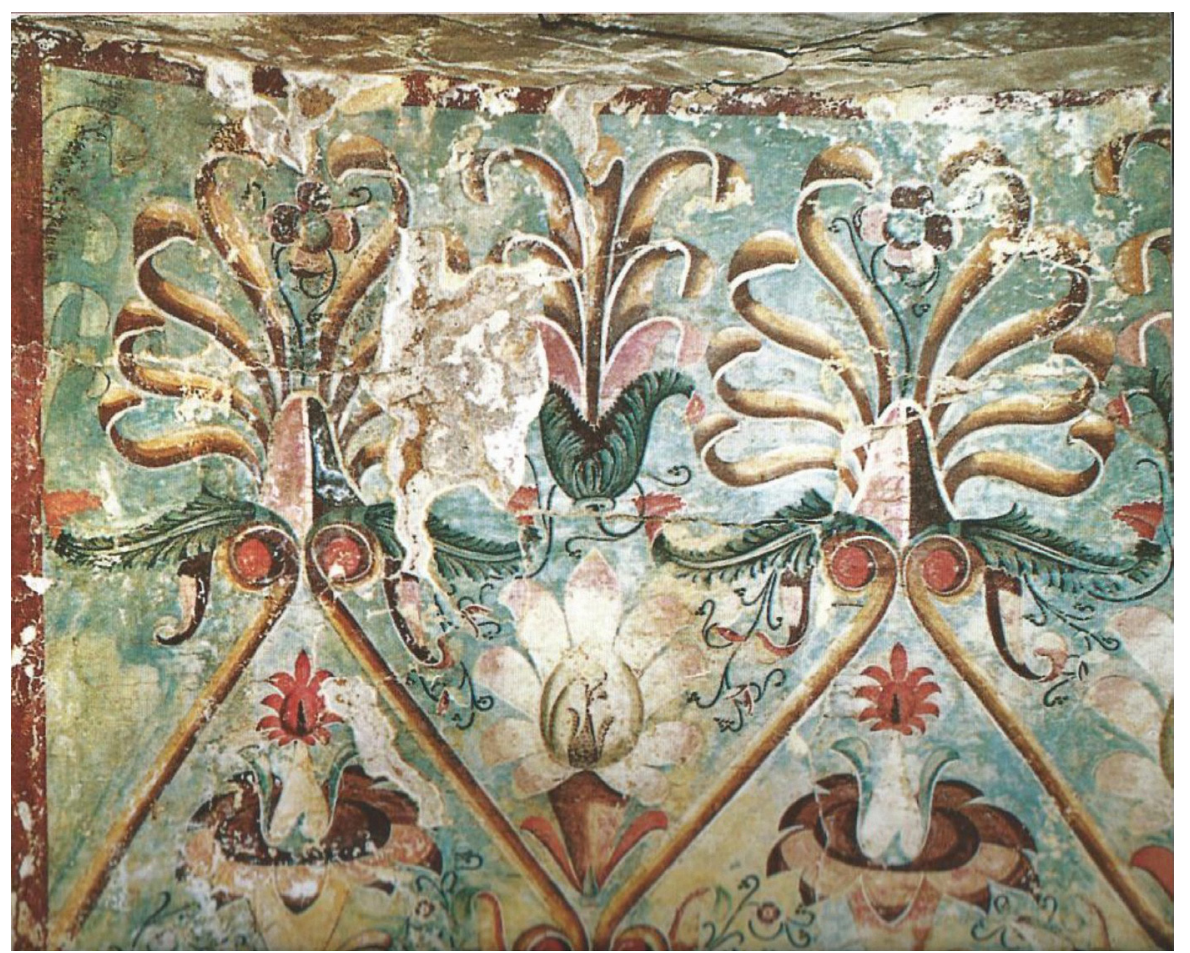

Figure 2

Mieza, peinture de l'antichambre de la tombe des palmettes. Photo : V. Vassal.
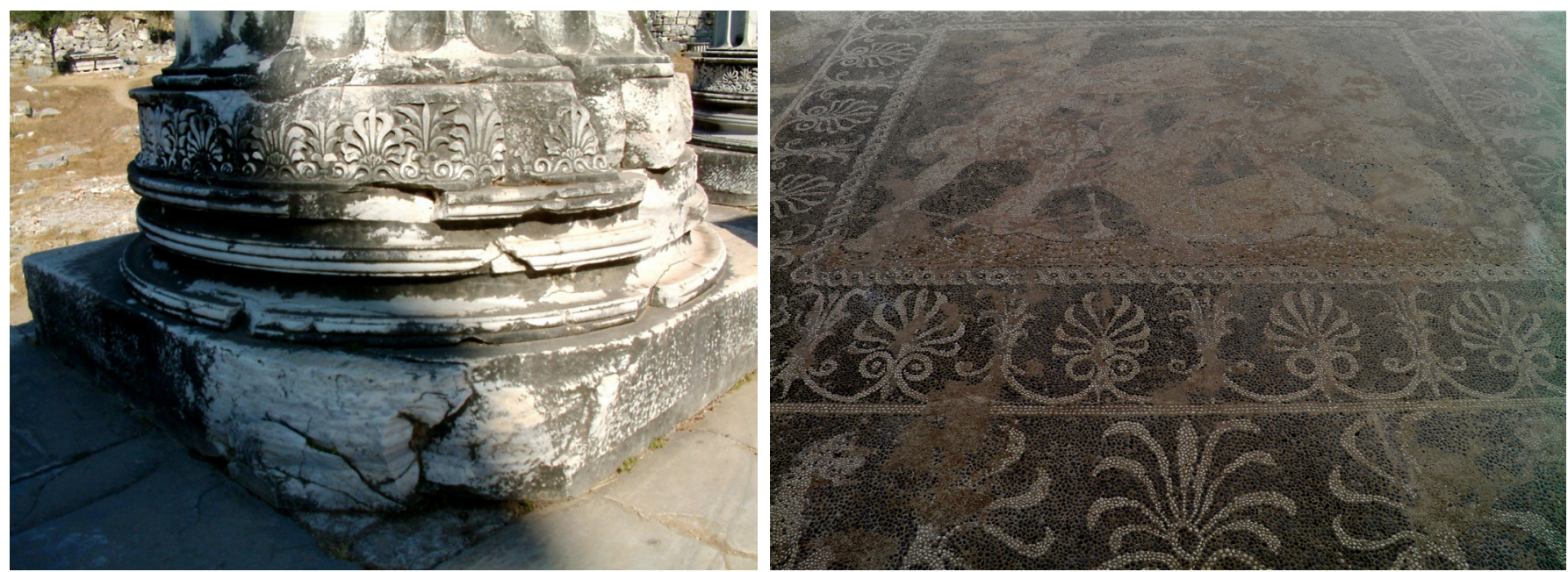

L'aspect naturaliste, très présent sur les mosaïques hellénistiques semble se transformer à l'époque impériale. Le lotus conserve-t-il quelque chose de la vision florale antérieure, lorsqu'il orne les pavements des provinces romaines ?

\section{Les scènes nilotiques}

Dans la maison du Faune à Pompéi, la mosaïque de la Bataille d'Alexandre et de Darius décorait une exèdre. Le seuil, délimité par deux colonnes corinthiennes, était orné d'une mosaïque, composée de plusieurs panneaux illustrant des scènes de vie sur le Nil, datés de la fin du II ${ }^{\mathrm{e}}$ siècle avant J.-C. Ces derniers ont été largement décrits et nous ne nous intéresserons ici qu'à la représentation des fleurs de lotus.

L'illustration qui en est faite semble décrire visuellement les différents stades du cycle de vie de la fleur. Ainsi on remarque à droite de l'un des panneaux ${ }^{5}$ (Fig. 1) un bouton épais, de forme conique, protégé par des sépales (qui semble

5 (MN, Naples, 9990).

Figure 3

Didymes, base d'une colonne du temple. Photo : V. Vassal.

Figure 4

Pella, bordure de la mosaïque de

l'Amazonomachie. Photo: V. Vassal. 
être au nombre de quatre). L'ensemble de ces sépales, de couleur vert amande, forme le calice et le bourgeon est rose pâle. Ce bouton est porté par un long pédoncule qui l'élève au-dessus de l'eau. La fleur est légèrement penchée vers la gauche. À côté d'elle, une autre excroissance qui contient en germe une seconde fleur suit la même inclinaison. Un petit peu plus bas sur la mosaïque, une fleur éclose et partiellement fanée laisse apparaître une rangée de cinq pétales alternativement rouge et rose. À l'origine la fleur devait porter plusieurs rangées de pétales extérieurs et intérieurs formant ainsi une double corolle. Au centre, un nombre important d'étamines prend naissance autour du réceptacle de forme conique. Une autre fleur semblable décore le centre de la scène. Quatre carpophores $^{6}$ sont dépourvus de pétales et portent un certain nombre d'alvéoles à ouverture circulaire. Chacun de ces orifices est censé comporter un fruit, un akène. Signalons la façon dont ces réceptacles ont été traités quant au choix des couleurs ; deux ont la partie extérieure verte, et la face supportant les fruits de couleur rose clair ; les deux autres présentent un ciboire vert/ brun et la partie alvéolaire est rouge. Cela correspond à la fin du cycle de vie de la plante. La fleur s'est fanée, les pétales et les étamines sont tombés, seul le réceptacle au sommet du pédoncule continue à mûrir afin de libérer les fruits. Chez les Égyptiens, l'association du lotus et de l'immortalité est sans doute liée à la longévité exceptionnelle de ses graines, capables de rester en dormance durant des siècles avant de germer lorsque les conditions sont propices. On sait d'après les textes ${ }^{7}$ que les habitants du Delta se nourrissaient de ces graines (Amigues 1995 : 51-52 ; $2007: 101)^{8}$. Les Grecs nommaient le lotus « fève d'Égypte » et Théophraste le décrit très précisément :

«La fève d'Égypte pousse dans les marécages et les lacs. Sa tige atteint au maximum quatre coudées de longueur sur un doigt d'épaisseur ; elle ressemble à un roseau tendre et sans nœuds, mais présente intérieurement des interstices répartis sur tout son diamètre comme les rayons de cire. Cette tige est surmontée de la tête qui ressemble un peu à un petit nid de guêpes arrondi. Chacune des alvéoles contient une fève légèrement proéminente; on en compte trente au maximum. La fleur est le double d'un pavot et sa couleur un rosé saturé. La tête se dresse au-dessus de l'eau. À côté de chacune des fèves poussent de grandes feuilles de dimensions égales à celles d'un feutre thessalien, qui ont une tige identique à celle des fèves. Quand on brise chaque fève, on en voit bien la partie amère, contournée, d'où naît la feuille en forme de feutre » (Theophr. h. plant. IV, 8, 7).

La présence de cette fleur en Égypte ne semble pas être antérieure à l'époque perse (Woenig 1971 : 34-44 ; Germer 1985 : 39-40). Les éléments d'architecture comme les colonnes lotiformes pourraient être liés à l'image du lotus blanc (Nymphea lotus L.) indigène en Égypte (Amigues 1995 : 51). Il s'agirait d'une variété à fleurs blanches de l'espèce Nymphaea caerulea (Amigues 2010 : 164 note 118).

6 Cette partie est aussi comparée à un ciboire qui donnera naissance au mot « ciborion » pour désigner le fruit.

7 Hdt. II, XCII : « Il y a une autre espèce de lis, ressemblant aux roses, et qui croît aussi dans le Nil. Son fruit a beaucoup de rapport avec les rayons d'un guêpier : on le recueille sur une tige qui sort de la racine, et croît auprès de l'autre tige. On y trouve quantité de grains bons à manger, de la grosseur d'un noyau d'olive : on les mange verts ou secs. »

8 Voir Grimaldi 2018: 5 : « Carbonized fruits of N. nucifera have been recovered from a late $4^{\text {th }}$ century BC necropolis at Salamis in Cyprus. Desiccated seeds of sacred lotus have also been found at Berenike (Egypt) and dated to the $1^{\text {st }}-2^{\text {nd }}$ century $\mathrm{AD} »$. 
Dans les peintures égyptiennes, il n'est pas rare d'observer des fruits à différents stades de maturité, comme sur l'un des fragments provenant de la chapelle tombale de Nebamon ${ }^{9}$ (Fig. 5) (Strudwick 2006 : 176 ; Middeleton - Uprichard 2008). Différentes espèces d'arbres fruitiers sont représentées comme les palmiers-dattiers, les figuiers-sycomores ou les palmiers doums. Au centre du fragment, un étang rectangulaire présente une faune marine, des canards et des lotus (probablement la variété de Nymphea caerulae, comme le suggère l'aspect pointu des pétales de la fleur, bien différent de ceux du Nelumbo nucifera). L'eau est de couleur bleue ; de fines lignes horizontales marquent l'ondulation et aucune ombre n'est apparente. Les fleurs et les oiseaux sont dessinés de profil sur le fond azur (Fig. 6). Il en est de même, pour ne citer qu'un seul exemple, de l'une des parois peintes de la tombe de Menna (TT69) à Thèbes ${ }^{10}$. Une scène de chasse dans un marais y est finement traitée. Il s'agit d'un thème classique qui n'a pratiquement pas évolué depuis l'Ancien Empire. Un homme se tient debout sur une barque en papyrus et roseaux ligaturés dont on remarque les extrémités en bouton. La partie inférieure de la paroi laisse entrevoir les eaux fourmillant de vie animale. Les canards nagent, se posent au milieu des lotus ouverts, ou en bouton. L'eau est symbolisée par des lignes brisées, disposées horizontalement et de couleur bleue (Fig. 7).

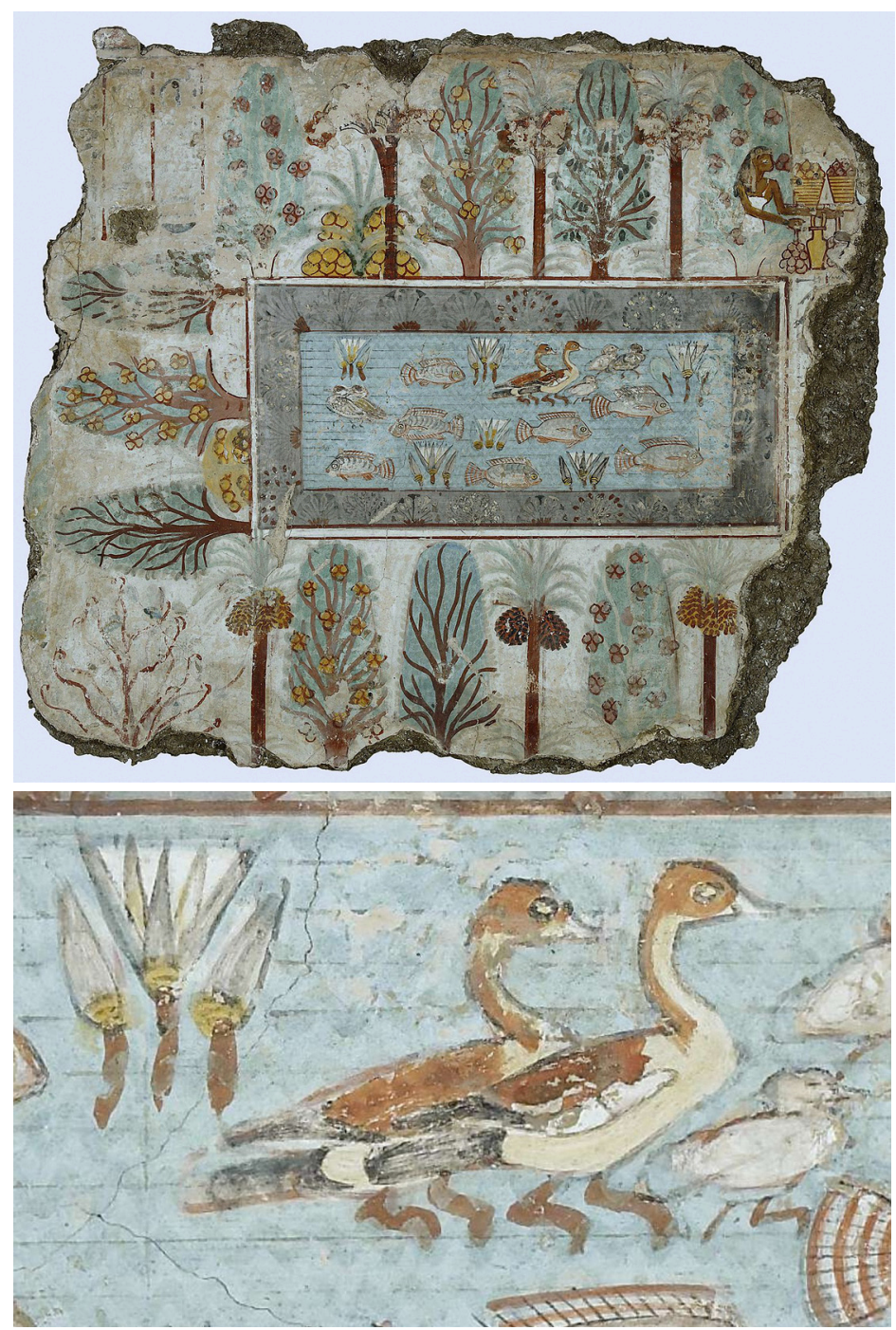

9 Daté de la XVIII ${ }^{\mathrm{e}}$ dynastie et conservé au British Museum (ES. 177).

10 Daté de la XVIII ${ }^{e}$ dynastie. Pour l'ensemble du décor peint de la tombe voir Hartwig 2013.
Figure 5

Thèbes, fragment provenant de la chapelle tombale de Nebamon. Photo : V. Vassal.

Figure 6

Thèbes, détail d'un fragment provenant de la chapelle tombale de Nebamon.

Photo : V. Vassal. 


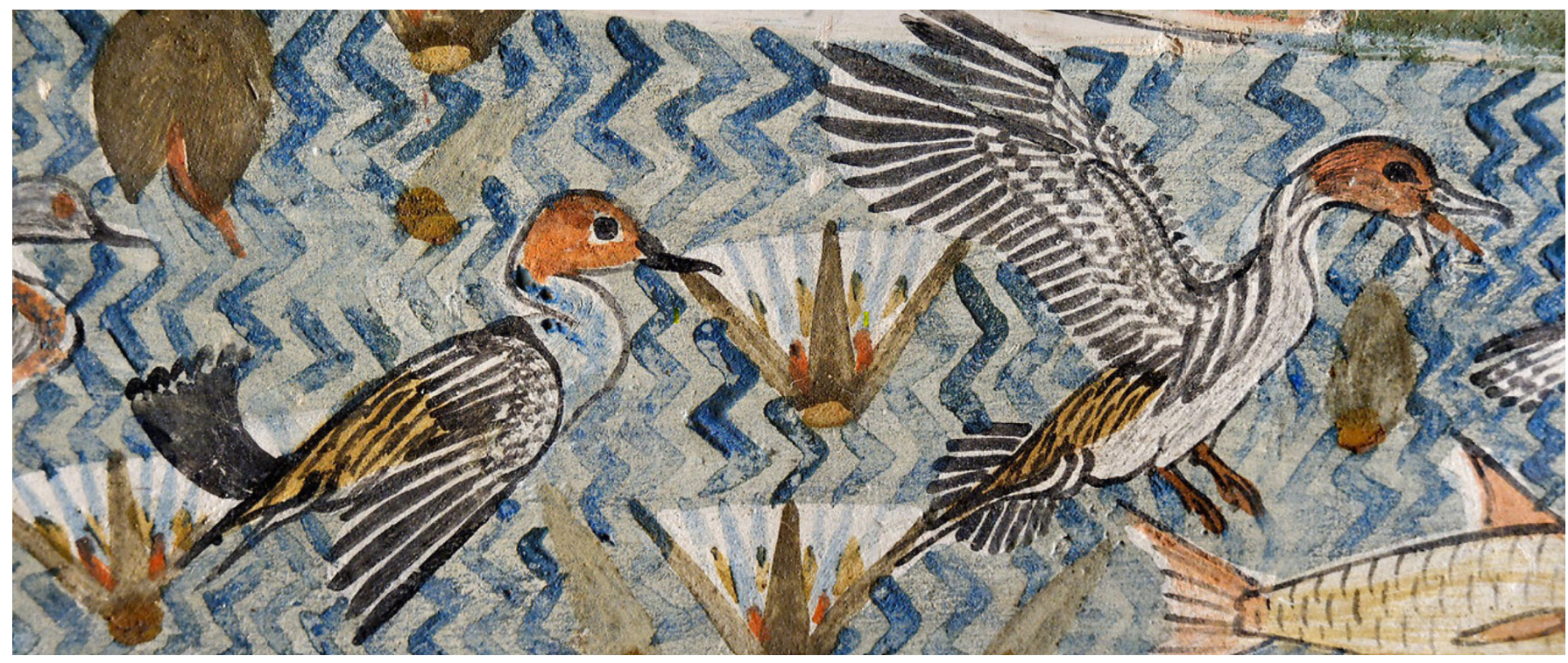

Figure 7

Thèbes, détail de la paroi de la tombe de Menna (TT69). Photo : V. Vassal.

Figure 8

Préneste, détail de la Mosaïque Barberini. Photo : V. Vassal.
Sur les décors de mosaïques, à l'époque hellénistique, nous retrouvons presque toujours, des végétaux figurés de façon plus ou moins schématisée, mais empreint d'un grand naturalisme. Les lotus peuvent être identifiés, cette fois, comme des nelumbo nucifera, représentés en bouton, en fleur, sans étamine... C'est le cas, notamment, sur la mosaïque Barberini à Préneste, où les divers stades de la vie de la fleur défilent, de même que sur le panneau provenant de la maison du Faune, cité précédemment. Le lotus apparaît parfois de face, ses pétales déployés. Le nelumbo se compose alors de plusieurs corolles, dont les pétales arborent des dégradés de couleur allant du rose foncé au rose clair (Fig. 8). Ce traitement n'est pas sans rappeler celui du fragment de la mosaïque des bains de Canope

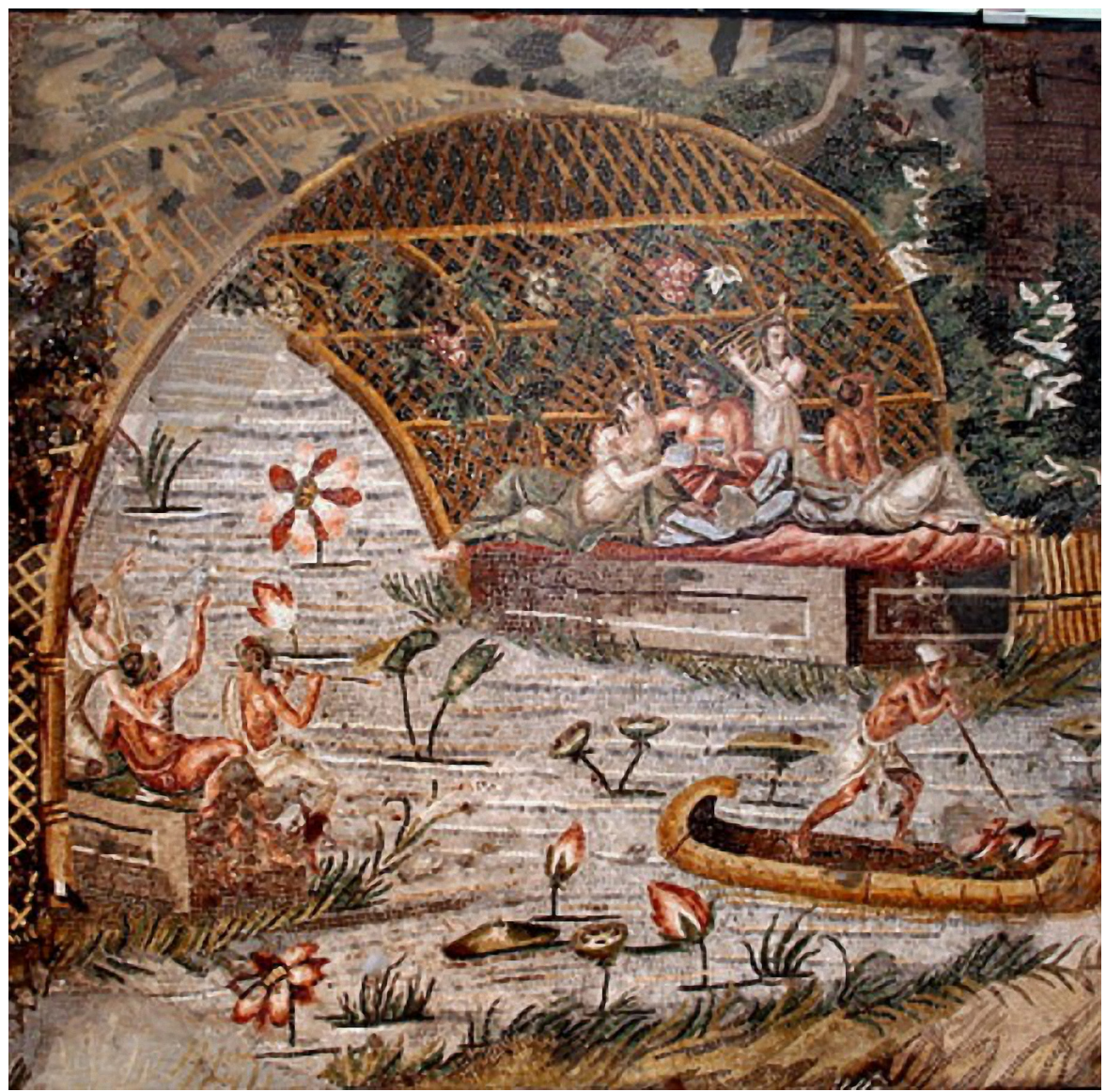


où un large nélumbo à deux corolles se déploie, vue du dessus, en composition circulaire (Daszewski 1978 : 125-126 fig. 113-114; Guimier-Sorbets 2009 : 650 fig. 8 ; Guimier-Sorbets - Redon 2017 : 149, 168 fig. 33 ; 2019a : 108-109 fig. 110, 226 ; Vassal 2018 : 74-75 fig. 9a). À Préneste, le réceptacle n'est plus visible, seules les fleurs s'épanouissent au bout de longs et fins pédoncules, ils sont disposés en plans successifs afin de créer un effet de profondeur. Le même traitement est présent sur la mosaïque de Privernum, daté entre le $\mathrm{II}^{\mathrm{e}}$ et $\mathrm{I}^{\mathrm{er}}$ avant J.-C. dont le seuil à décor nilotique ornait le triclinium d'une domus. La scène est savamment orchestrée par des éléments d'architecture, où des pygmées évoluent au milieu d'une faune ordinaire évoquant le Nil : canards, poissons, crocodiles, oiseaux.

À Privernum les motifs végétaux prennent une importance indéniable au vu de leurs répétitions sur le seuil et dans les compartiments du pavement qui décorait le triclinium (Cancellieri 2010 : 86, 89 fig. 23). Le premier motif à considérer est la fleur en bouton; elle se situe à peu près au centre du panneau, juste à droite du crocodile en train de croquer la jambe de l'un des pygmées (Fig. 9). Le bouton floral ovale et pointu apparaît enveloppé de deux sépales bleu vert.

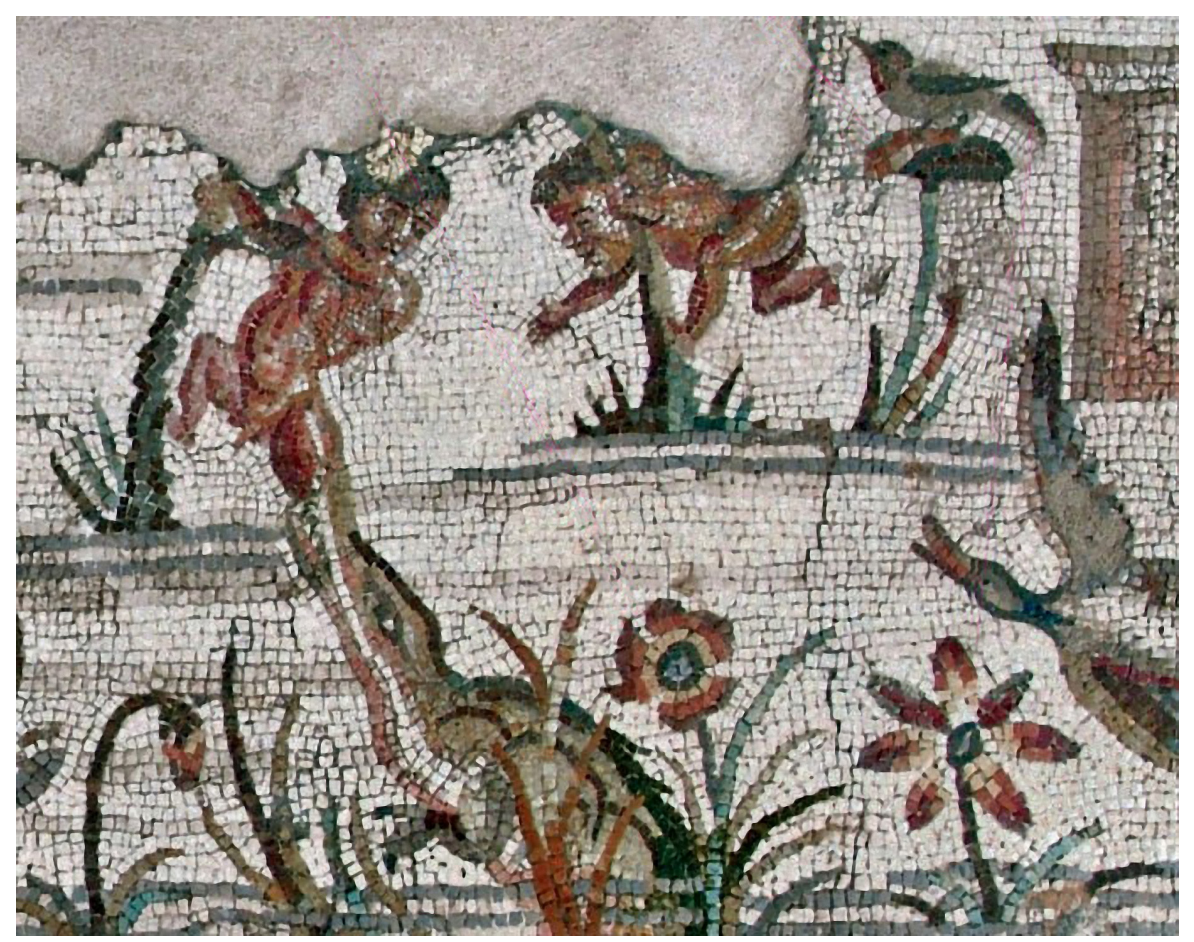

La corolle placée à sa droite est plus avancée dans son développement. Les pétales s'élargissent progressivement ; ils ne sont pas à leurs extensions maximales mais ils révèlent néanmoins le cœur de la fleur et la couleur jaune suggère les étamines serrées contre le réceptacle. Le lotus est tourné vers le spectateur, il n'est plus représenté de profil. Enfin le troisième nélumbo est parfaitement ouvert, étalant de plus en plus ses pétales, libérant probablement le pollen afin d'attirer les insectes pollinisateurs. Les hautes feuilles protectrices orbiculaires à bords relevés qui accompagnent généralement les nélumbos sont ici éloignées de l'inflorescence. Ces dernières sont réalisées à l'aide de tesselles de calcaire de différentes couleurs afin de matérialiser les courbes des limbes et accentuer les effets de perspective illusionniste. Tout comme sur les pavements de la maison du Faune à Pompéi et de Preneste, l'utilisation de tesselles de verre est également à souligner (Boschetti 2011 : 65, 69 fig. 12, 71-72 fig. 15). Pour les propriétaires, la représentation de ces fleurs pouvait évoquer, le présent,
Figure 9

Privernum, détail du seuil nilotique. Photo : V. Vassal. 
Figure 10

Nîmes, Détail de l'un des panneaux de la mosaïque de Bellérophon. Photo : Musée de la Romanité - Ville de Nîmes. le passé et le futur en raison de la présence simultanée de la plante en bouton, de la fleur et du fruit, marquant ainsi, par analogie, le caractère éphémère de la vie humaine ${ }^{11}$.

Sur les quelques exemples que nous avons cités, le lotus est très souvent accompagné de couple de canards, mais aussi d'oies du Nil, d'oiseaux et d'autres espèces marines. Le canard reste cependant dans l'univers mental, un gibier, lié à l'aucupium, la capture des oiseaux dont les Romains étaient très friands (Vendries 2009 : 119-140). Il n'en demeure pas moins un animal familier de la faune et des représentations nilotiques. Les canards nagent côte à côte, sont nichés dans une fleur de lotus, dans le creux d'une feuille, portent à leur bec une tige de lotus ${ }^{12}$.

En Gaule, l'un des rares exemples présentant des végétaux ressemblant à des feuilles de lotus, associés à des canards, décore l'un des panneaux de la mosaïque de Bellérophon à Nîmes datant du III ${ }^{\mathrm{e}}$ siècle après J.-C. (Fig. 10). J. Aymard dans son article de 1953 décrit très précisément les oiseaux aquatiques, qu'il identifie comme des canards sauvages (Aymard 1953 : 256). En ce qui concerne

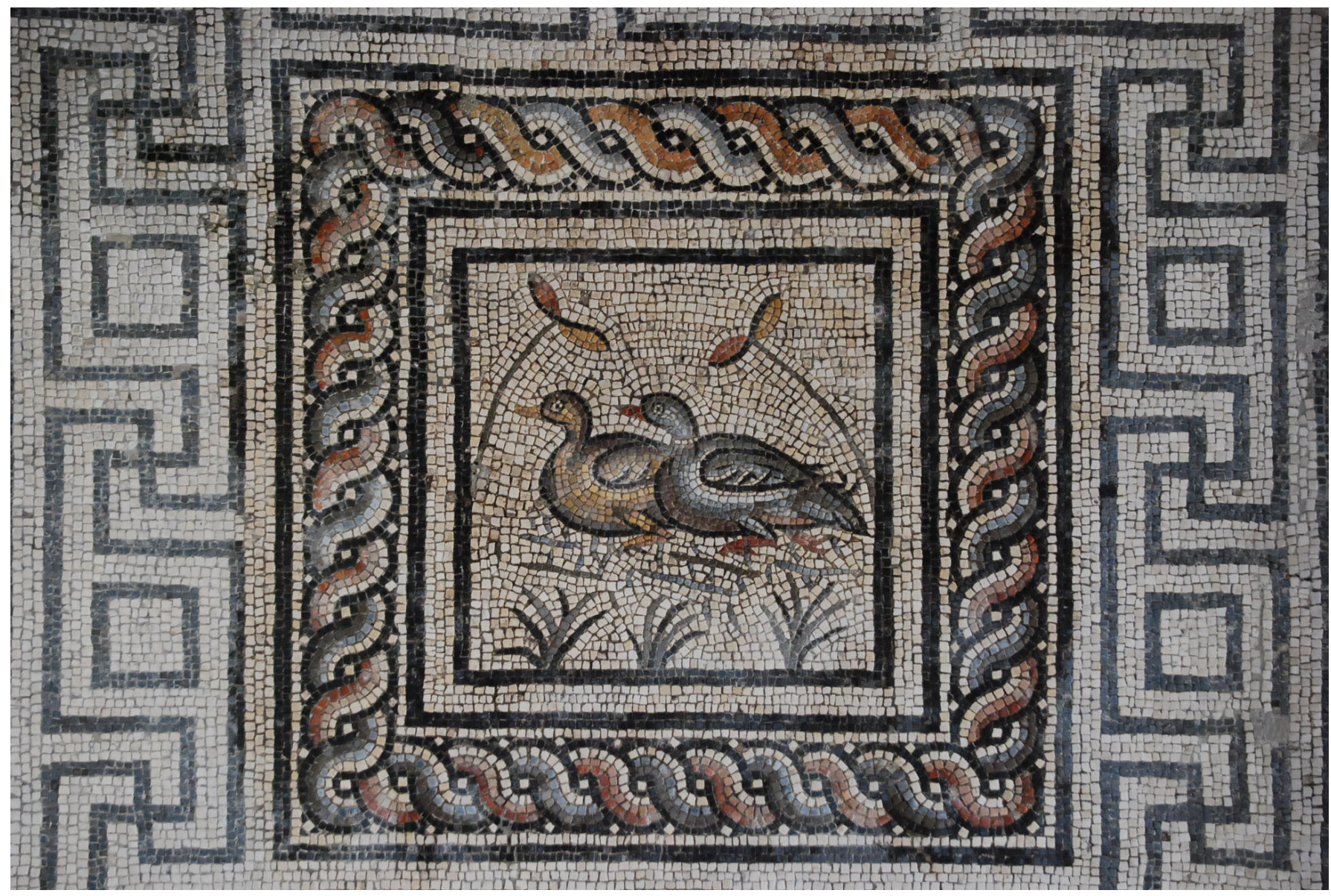

les deux végétaux de la partie supérieure, il précise que : « [...] se dressent deux tiges hautes et flexibles, sommées d'une large feuille hélicoïdale. » (Aymard 1953 : 255). À aucun moment il n'identifie ces deux végétaux, à des feuilles de lotus. Il est vrai que nous sommes dans un contexte régional, bien éloigné des thématiques nilotiques très prisées en Italie. Cependant, le thème

11 Il peut également s'agir d'une évocation du temps cyclique et d'un rapprochement établi entre la vie de la fleur et la vie humaine. Pour la conception circulaire du temps, voir Chenet $2000: 47-51$.

12 Aymard $1953: 265$ et notes : « [...] développant ainsi leur pouvoir grâce à la manducation de végétaux prophylactiques ». 
de cette mosaïque peut être rapproché du décor provenant du châle de Sabine, toile copte de laine rouge à décor de tapisseries, découvert à Antinoé, et daté entre les $\mathrm{IV}^{\mathrm{e}}$ et $\mathrm{V}^{\mathrm{e}}$ siècles après J.-C. (Fig. 11) ${ }^{13}$. Des petits putti s'ébattent dans un paysage nilotique composé de touffes de lotus roses entre lesquels évoluent des couples de canards. Le médaillon central présente Bellérophon aux prises avec la Chimère (Calament - Durand 2013 : 374-387). Parmi les pavements à sujets mythologiques qui nous sont parvenus, ceux qui illustrent l'histoire de Bellérophon sont assez peu nombreux. Nous connaissons au $\mathrm{IV}^{\mathrm{e}}$ siècle avant

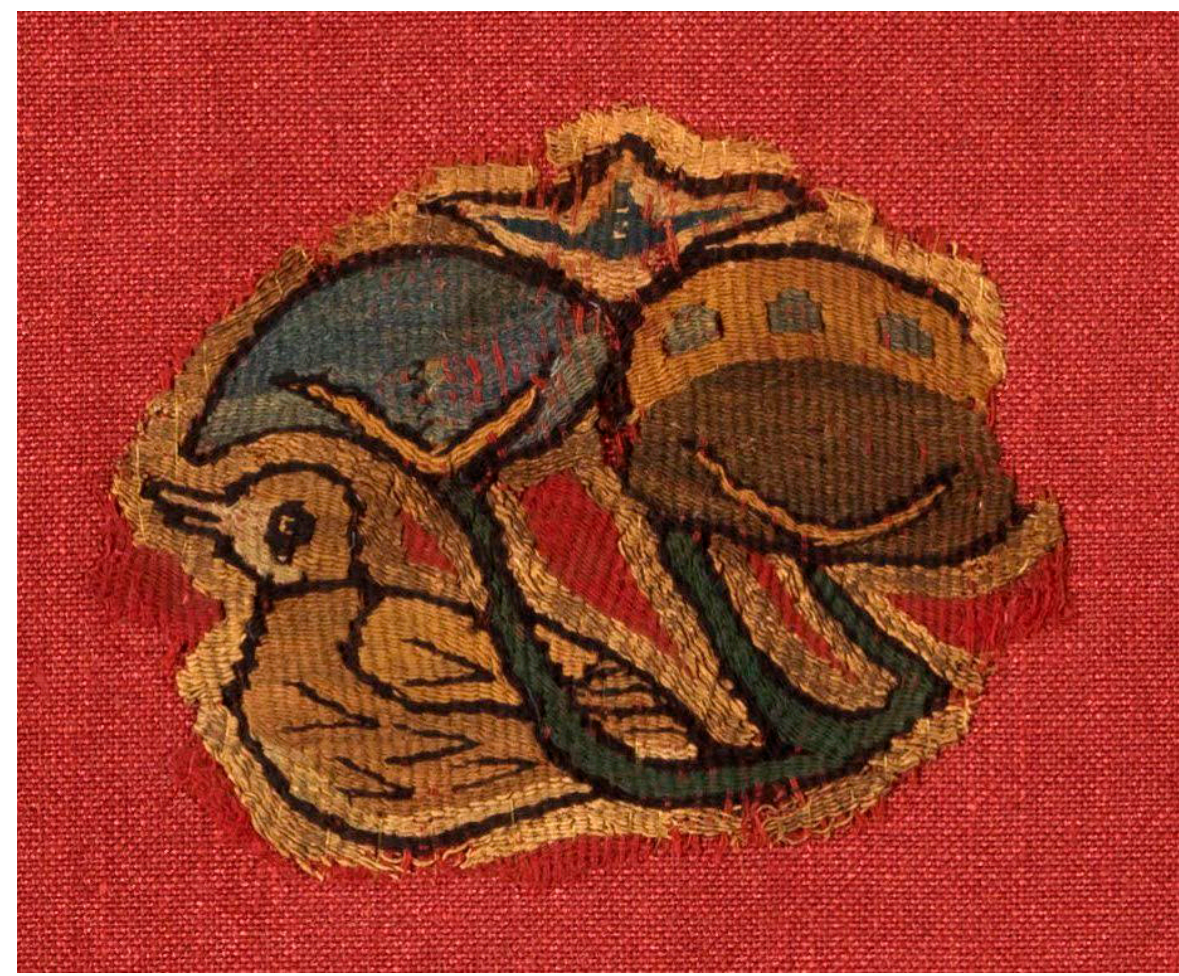

J.-C, le pavement d'Olynthe (Salzmann 1982 : nº79 11, 23, 99-100 pl.15.2) puis le mythe semble disparaître pour resurgir dans le courant des $\mathrm{II}^{\mathrm{e}}-\mathrm{III}^{\mathrm{e}}$ siècles en Occident : Autun, Reims, Avenches et Herzogenbuchsee (Delbarre-Bärtschi 2014 : 108, 205 pl. 15, 247 pl. 35,4) Ravenne, Conimbriga, Lullingstone, Parndorf (Toynbee 1955 : 91-97 ; Amandry 1956 : 155 - avec mention de la bibliographie antérieure) en Orient à Constantinople et Palmyre (Gawlikowski 2005 : 1293-1304). D'après les éléments stylistiques identifiés, seul l'exemple de Nîmes présente un couple de canards accompagné de feuille de lotus, comme sur les médaillons entourant Bellérophon sur le châle de Sabine. Les analyses effectuées sur des fragments de tissu mettent en évidence des colorants utilisés par les Égyptiens : la garance, l'indigo ou la gaude (Rutschowscaya 2004 : 59). Il semble donc que notre panneau nîmois évoque un paysage nilotique extrêmement stylisé, dont l'origine nous échappe. Mais les thématiques des panneaux associés au mythe de Bellérophon semblent toutes avoir un lien. À Nîmes, Bellérophon et les Saisons peuvent s'interpréter dans le sens prophylactique ou eschatologique. La Chimère terrassée par Bellérophon représente l'allégorie du bien contre le mal ou la mort domptée qui peut être revendiquée aussi bien par la Rome impériale que par la chrétienté. L'évocation du Nil, c'est le renouveau et la promesse d'une vie meilleure.

13 Les fragments sont dispersés dans trois musées (musée du Louvre, musée des tissus de Lyon, musée des Beaux-Arts de Lyon). Un ensemble de quatorze motifs a été mis en vente aux enchères en 2013. Ces motifs sont aujourd'hui au Louvre Lens.
Figure 11

Antinoé, fragment du châle de Sabine. Photo : V. Vassal. 
Figure 12

Tabgha, détail de la mosaïque de l'église de la multiplication des pains. Photo : V. Vassal.

Dans l'église de la multiplication des pains à Tabgha, datée du V'e siècle après J.-C. (Versluys 2002 : 228-230), la mosaïque est agrémentée d'oiseaux aquatiques entourés de larges et grands nélumbos stylisés (Fig. 12). Les motifs végétaux représentés ne sont pas réalistes et sont traités dans un tout autre esprit que ceux des mosaïques précédemment étudiées. Les fleurs, les fruits et les feuilles semblent disproportionnés ; il n'y a pas d'éléments de paysage. Ni la couleur, ni le traitement de la surface ne trahit la présence de l'eau, pourtant un nilomètre rappelle le Nil. Le mosaïste remplit l'espace à l'aide de motifs de fleurs, de canards, d'oiseaux et subordonne ainsi, la composition à l'effet décoratif. Ce traitement vient probablement de l'Orient.

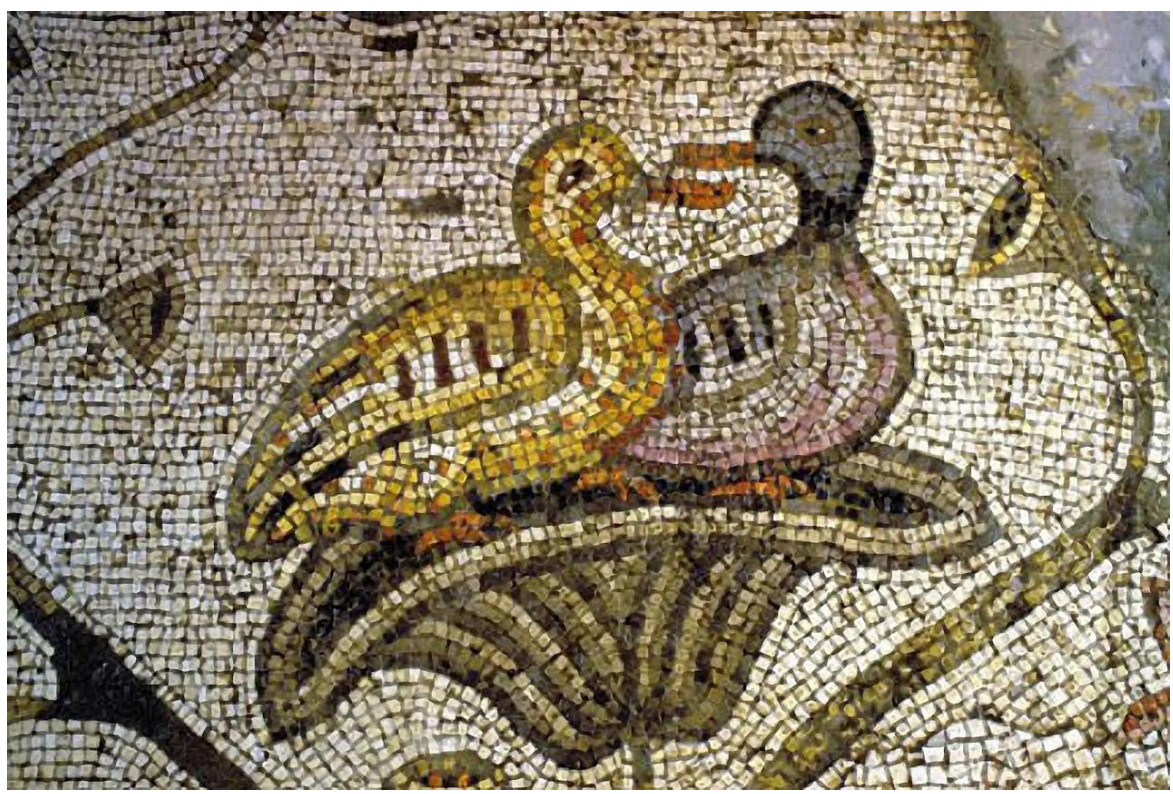

Nous pourrions multiplier les exemples et approfondir notre propos, mais les quelques remarques exprimées montrent l'intérêt que portaient les Romains aux scènes nilotiques et particulièrement à la faune et à la flore du Nil.

L'exemple du lotus est prégnant puisqu'il est omniprésent sur toutes les mosaïques à décor de scènes nilotiques. Mais étonnamment, les fleurs sont toujours des Nelumbos nucifera et jamais des Nymphea caerulea (bleu), ni même des Nymphea lotus bien identifiés dans l'iconographie égyptienne ${ }^{14}$. Si les mosaïstes ayant conçu les pavements d'époque hellénistique - comme ceux de Pompéi et Préneste - étaient originaires d'Alexandrie (Meyboom 1995 : 91 95), on s'étonne de ne jamais voir de confusions entre les différentes fleurs. Sauf si les Nymphea caerulea avaient, à cette époque, totalement disparu du paysage égyptien. Or Athénée (Athen. deipn. XV, 677) rapporte qu'au cours du IIe siècle après J.-C., on tressait à Alexandrie des couronnes de lotus bleu. Comment est-il possible que les mosaïstes alexandrins ou de formation alexandrine n'aient pas reproduits les variétés de fleurs aux pétales étroits Nymphea caerulea. Ces fleurs n'étaient-elles plus en vogue, limitées à des cultures dans des jardins privés ?

Si l'on considère maintenant la représentation de l'eau sur les mosaïques nilotiques, on remarque que le traitement est réalisé à l'aide de fines lignes de tesselles en dégradé de couleur, sur un fond gris-bleu, bleu-clair, tout du moins pour les œuvres de la période hellénistique. Ce traitement est très proche des exemples des tombes de Menna et Nebamon où la transparence, les frissonnements de l'eau sont dépeints, pour l'un, avec de fines lignes parallèles

14 Il n'est pas possible de voir des Nelumbo nucifera sur les œuvres de l'Égypte pharaonique puisque ces derniers ont été introduits par les Perses. 
gris-bleu, et pour l'autre avec des lignes brisées horizontales bleu foncé. La comparaison entre mosaïques et peintures est évidemment rendue délicate par le décalage chronologique qui les sépare.

Nous avons évoqué très brièvement le décor chrétien de l'église de Taghba où la couleur de l'eau a totalement disparue. Lorsque l'on se tourne vers la partie orientale de l'Empire, cette caractéristique semble être un principe. Le Nil et ses bienfaits sont caractérisés par les végétaux stylisés et la faune; cela donne aux scènes, un aspect très décoratif mais plus du tout naturaliste.

Ces quelques remarques montrent que les mosaïstes ont cherché à représenter avec grande précision la flore égyptienne, tout du moins à l'époque hellénistique. L'aspect naturaliste a disparu au fil du temps, mais la symbolique devait toujours être présente en fonction du lieu où se situait la mosaïque dans la maison, de l'origine du commanditaire, du contexte chronologique et géographique. Les nélumbos ne peuvent être dissociés des scènes nilotiques qui symbolisaient le renouveau, la luxuriance ; ces scènes souvent à fonction apotropaïque, pouvaient être également interprétées, comme nous le mentionnions précédemment, d'un point de vue eschatologique.

\section{Bibliography - Kaynaklar}

Amandry 1956

Amigues 1995

Amigues 2007

Amigues 2010

Athen. deipn.

Aymard 1953

Boardman 1998

Boschetti 2011

Calament - Durand 2013

Cancellieri 2010

Chenet 2000

Daszewski 1978

Delbarre-Bärtschi 2014

Gawlikowski 2005

Germer 1985

Ginouvès 1993

Grimaldi et al. 2018

Guimier-Sorbets 2009
P. Amandry, "Bellérophon et la chimère dans la mosaïque antique”, RA 48, 155-161.

S. Amigues, "Les plantes d‘Égypte vues par les naturalistes grecs", J. Leclant (ed.), Entre Égypte et Grèce. Actes du $5^{\text {ème }}$ colloque de la Villa Kérylos à Beaulieu-sur-Mer du 6 au 9 octobre 1994, Paris, 1-67.

$\mathrm{S}$. Amigues, "L'exploitation du monde végétal en Grèce classique et hellénistique. Essai de synthèse”, Topoi $15 / 1,75-125$.

S. Amigues, Théophraste Recherches sur les plantes. A l'origine de la botanique, Paris.

Athénée, Deipnosophistae, trad. A.-M. Desrousseaux, Paris 2002.

J. Aymard, "La mosaïque de Béllérophon à Nîmes”, Gallia 11,2, 249-271.

J. Boardman, "Reflections on the Origins of Indian Stone Architecture", Alexander's Legacy in the East Studies in Honor of Paul Bernard, BAsInst 12, 13-22.

C. Boschetti, "Vitreous Materials in Early Mosaics in Italy: Faience, Egyptian Blue, and Glass", JGS 53, 59-91.

F. Calament - M. Durand (dir.), Antinoé à la vie à la mode. Visions de l'élégance dans les solitudes, Lyon.

M. Cancellieri, "Case e mosaici a Privernum. Parte II: La domus della Soglia nilotica”, Musiva \& Sectilia 4, 63-141.

F. Chenet, Le Temps. Temps cosmique, temps vécu, Paris.

A.W. Daszewski, "Some problems of early mosaics from Egypt”, Das ptolemaische Egypten, Berlin-Mayence, 123-136.

S. Delbarre-Bärtschi, Les mosaïques romaines en Suisse. Avec un complément de l'inventaire de Victorine von Gonzenbach, publié en 1961, Bâle.

M. Gawlikowski, "L'apothéose d'Odeinat sur une mosaïque récemment découverte à Palmyre", CRAI 149, 4, 1293-1304.

R. Germer, Flora des pharaonischen Ägypten, Mayence.

R. Ginouvès, La Macédoine, de Philippe II à la conquête romaine, Paris.

I. M. Grimaldi - S. Muthukumaran -G. Tozzi - A. Nastasi - N. Boivin - P. J. Matthews - T. van Andel, "Literary Evidence for Taro in the Ancient Mediterranean: A chronology of Names and Uses in a Multilingual World", PLoS ONE 13/6, 1-23.

A.-M. Guimier-Sorbets, "Scènes nilotiques : expression de l'abondance et vision de l'autre", Hairy I. (ed.), Du Nil à Alexandrie, histoire d'eaux, catalogue de 1'exposition Laténium, Alexandrie : Centre d'Etudes Alexandrines, 644-663. 
Guimier-Sorbets 2013

Guimier-Sorbets 2019a

Guimier-Sorbets 2019b

Guimier-Sorbets - Redon 2017

Hdt.

Janick 2002

Li et al. 2014

Meyboom 1995

Middeleton - Uprichard 2008

Morat 2004

Rutschowscaya 2004

Salzmann 1982

Strudwick 2006

Theophr. h. plant.

Toynbee 1955

Vassal 2018

Vendries 2009

Versluys 2002

Versluys - Meyboom 2000

Whitehouse 2003

Wiegand 1941

Woenig 1971
A.-M. Guimier-Sorbets, “Les plaisirs de la vie dans l'Égypte gréco-romaine : iconographie d'une mosaïque de Thmouis", D. Lauritzen - M. Tardieu (dir.), Le voyage des légendes. Hommages à Pierre Chuvin., Paris, 141154.

A.-M. Guimier-Sorbets, avec la collaboration d'A. Guimier, N. Morand et D. Weidmann, Mosaïques d'Alexandrie Pavements d'Égypte grecque et romaine, Alexandrie.

A.-M. Guimier-Sorbets, "Les scènes nilotiques sur les mosaïques aller et retour entre Orient et Occident", C. Arnould-Béhar - V. Vassal (dir.), Art et archéologie du Proche-Orient hellénistique et romain : les circulations artistiques entre Orient et Occident, volume 2, Oxford, 101-110.

A.-M. Guimier-Sorbets - B. Redon, "The floors of the Ptolemaic Baths of Egypt. Between Technique and Aesthetics”, B. Redon (ed.), Collective Baths in Egypt 2. New discoveries and perspectives, Etudes Urbaines 10, Institut français d'archéologie orientale, Cairo, 139-170.

Hérodote, Histoire, Livre II, trad. Philippe-Ernest Legrand, Paris 2010.

J. Janick, “Ancient Egyptian Agriculture and the Origins of Horticulture”, Acta Horticulturae 583, 23-39.

Y. Li - T. Smith - P. Svetlana -J. Yang - J.-H. Jin - C.-S. Li, "Paleobiogeography of the lotus plant (Nelumbonaceae: Nelumbo) and its bearing on the paleoclimatic changes", Palaeogeogr. Palaeoclimatol. Palaeoecol 399, 284293.

P. G. P. Meyboom, The Nile Mosaic of Palestrina. Early Evidence of Egyptian Religion in Italie, Leyde.

A. Middleton - K. Uprichard (ed.), The Nebamun Wall Paintings: Conservation, Scientific Analysis and Display in the British Museum, London.

P. Morat, "Les guirlandes de Ramsès II”, P. Morat - G. Aymonin - J.-C. Jolinon (eds.), L'Herbier du monde : Cinq siècles d'aventures et de passions botaniques au Muséum d'histoire naturelle, Paris, 176-180.

M.-H. Rutschowscaya, Le châle de Sabine, Paris.

D. Salzmann, Untersuchungen zu den antiken Kieselmosaiken, Berlin.

N. Strudwick, Masterpieces of Ancient Egypt, Londres.

Théophraste, Recherche sur les plantes, Livres III et IV, trad. Suzanne Amigues, Paris 2003.

J. Toynbee, "Mosaïques au Bellérophon”, Gallia 13,1, 91-97.

V. Vassal, "Motifs géométriques et floraux dans les mosaïques Hérodiennes : regards croisés", C. ArnouldBéhar - V. Vassal (dir.), Art et archéologie du Proche-Orient hellénistique et romain : les circulations artistiques entre Orient et Occident, volume 1, Oxford, 69-78.

C. Vendries, "L'auceps, les gluaux et l'appeau. A propos de la ruse et de l'habileté du chasseur d'oiseaux", J. Trinquier - Chr. Vendries (dir.), Chasses antiques. Pratiques et représentations dans le monde grécoromain (IIIe s. av. -IVe s. apr. J.-C.). Actes du colloque international de Rennes, Rennes, 119-140.

M. J. Versluys, Aegyptiaca romana. Nilotic scenes and the Roman views of Egypt, Leiden, Boston.

M. J. Versluys - P. G. P Meyboom, "Les scènes dites nilotiques et les cultes isiaques. Une interprétation contextuelle", L. Bricault (ed.), De Memphis à Rome, Actes du 1er Colloque international sur les études isiaques, Poitiers, Leiden-Boston-Köln, 111-127.

H. Whitehouse, "The Nile Flows Underground to Cyprus : The Painted Water-Cistern at Salamis Reconsidered", D. Michaelides - V. Kassianidou - R. S. Merillees (eds.), Egypt and Cyprus in Antiquity : Proceedings of the International Conference, Nicosia, 252-259.

T. Wiegand, Didyma vol. 1, Berlin.

Fr. Woenig, Die Pflanzen im Alten Aegypten, Leipzig, 1886, Réimpr. 
